\title{
Gewässerverschmutzung durch Außenbordmotoren und deren Wirkung auf Fauna und Flora
}

\author{
DIETRICH LÜDEMANN \\ Bundesgesundheitsamt, Institut für Wasser-, Boden-und Lufthygiene, Berlin-Dablem
}

\begin{abstract}
Water pollution by outboard motors and its effects on fauna and flora. Cases of water pollution by outboard motors have been reported in America and Switzerland. The present studies have been sponsored by the German Federal Board of Health in co-operation with the Institute for Internal Combustion. Engines of the Technical Uaiversity of Berlin. We have conducted (a) motor brake tests, (b) tank tests and (c) tests in ponds, considering effects of dissolved engine gases on fishes and fish food organisms in relation to fuel consumption and test duration. Tests were made with 3 new, mixed-lubricated twostroke carburettor engines with underwater exhausts and a capacity of 6,20 and 40 PS, respectively, releasing a drift of oil, as well as petrol and its combustion products. Of special interest were hydrocarbons because of their poor biochemical decomposition in organisms and phenols in view of their toxicity in water. Lethal doses differed in the fishes (carps, trouts) tested. During pond tests these fishes turned out to be sensitive indicators for the degree of water pollution because accumulation of combustion products in the water can be tested very quickly in terms of fish flesh flavour. Experiments in aquariums containing a concentration of $1: 2000$ demonstrated that detrimental changes in the flavour of the fish flesh disappeared after some days exposure to clean freshwater. The pond experiments further revealed a significant reduction in the number of certain microorganisms. After termination of tests, the chemical and biological conditions improved increasingly within a few weeks due to self-purification of the water. Our investigations show clearly that, in waters serving as drinking water supplies, all boat activities must be avoided.
\end{abstract}

\section{EINLEITUNG}

Über die Verölung von Gewässern durch Außenbordmotoren liegen verhältnismäßig wenig Veröffentlichungen vor, die zudem vom wissenschaftlichen Standpunkt aus unterschiedlich zu bewerten sind, da oft nur motortechnische Fragen untersucht wurden. Allgemein kann aber festgestellt werden, daß in einigen Fällen in Amerika und der Schweiz bereits Gewässer durch den Betrieb von Außenbordmotoren erheblich verschmutzt worden sind, und daß sich dadurch ungünstige Auswirkungen auf die Organismenwelt des Wassers ergaben.

Die seinerzeit (1961) im Auftrag des amerikanischen Gesundheitsministeriums durchgeführten Tank- und Teichversuche behandelten hauptsächlich die Frage, inwieweit Fischfleisch durch Verölung von Gewässern im Geschmack geschädigt werden kann (English, McDermott \& Henderson 1961, English, Surber \& MCDermott 
1961). Ausführlichere Analysenbefunde, die unter verschiedenen motorischen Betriebsbedingungen ermittelt wurden, fehlen; ebenfalls wurde bei diesen. Versuchen die Einwirkung auf die Organismen kaum berücksichtigt. Das Verhältnis vom Schmieröl zu Brennstoff lag bei den einzelnen Teichversuchen zwischen $1: 17$ und 1:23. Als Anhaltswert wurde gefunden, daß ab 81 Brennstoff je $1000 \mathrm{~m}^{3}$ Wasser und Saison das Fischfleisch leidet. In weiteren Versuchen wurde ermittelt, daß die toxische Wirkung des verunreinigten Wassers schnell zeitlich abklingt.

Die sich für das Institut für Wasser-, Boden- und Lufthygiene bei der oben angeführten speziellen Fragestellung ergebenden chemischen und biologischen Aufgaben wurden mit dem von Herrn Professor Pflaum von der Technischen Universität Berlin aufgestellten motortechnischen Arbeitsplan koordiniert. Die Untersuchungen, für die 3 fabrikneue Motoren verschiedener Leistung (6,20 und 40 PS) und Bauart vorgesehen waren, wurden in 3 Abschnitten durchgeführt: (a) Bremsversuche, in denen abhängig von Motorleistung und Mischungsverhältnis Schmieröl: Brennstoff die anfallenden Abgas-, Ol- und Krackprodukte chemisch untersucht wurden. (b) Tankversuche, in denen die Löslichkeit von Auspuffgasen und Schmieröl eines Motors 1 : 50 (Mischungsverhältnis $1: 50$ ) im Tankwasser chemisch geprüft wurden. Gleichzeitig wurden mit diesen Wässern orientierende Toxizitäts- und Geschmacksteste mit Fischen durchgeführt. (c) Praxisnahe Teichversuche, in denen die unterschwellige Wirkung der gelösten Verbrennungsgase auf Fische (Karpfen, Forellen) und Kleinorganismen des Wassers in Abhängigkeit von Kraftstoffverbrauch und Versuchszeit biologisch festzustellen waren; auch die chemischen Befunde des Gewässers und seine äußere Beschaffenheit wurden laufend kontrolliert.

\section{ERGEBNISSE}

\section{Tankversuche}

Auf die vorwiegend motortechnischen Untersuchungen bei den Bremsversuchen möchte ich im Rahmen dieser Abhandlung nicht näher eingehen.

Für die sogenannten Tankversuche wurden durch Einleiten der Motorauspuffgase in einen eisernen wassergefüllten Tank von $2 \mathrm{~m}^{3}$ Inhalt zwei Versuchswässer verschiedener Konzentrationen in der Technischen Universität Berlin hergestellt.

Durch die "Tankversuche“, die mit Berliner Leitungswasser angesetzt wurden, sollten vor allem chemische Aussagen quantitativer und qualitativer Art über den Auswurf von Verbrennungsprodukten wie OI und leichtflüchtigen Stoffen bzw. über deren Löslichkeit im Wasser gewonnen werden. Neben der Feststellung des pH-Wertes, der äußeren Beschaffenheit, der Geruchs- und Geschmacksschwellenkonzentration wurden die gleichen Bestimmungen wie bei den Bremsversuchen durchgeführt. Auf ihre toxische Wirkung wurden die Wässer auch im Fischversuch geprüft und Geschmacksteste mit den Versuchstieren durchgeführt.

In Tabelle 1 sind die mit einem 20-PS-Motor und einem Schmieröl-Brennstoffgemisch von 1 : 50 ermittelten Analysenwerte zweier Versuchswässer mit den Konzentrationen von $1: 1000$ und $1: 2000$ (verbrauchter Brennstoff/Wasser) zusammengestellt. 
Tabelle 1

Analysenwerte zweier Versuchswässer in Tankversuchen

\begin{tabular}{|c|c|c|c|}
\hline Brennstoffverbrauch/Wasser & & $1: 1000$ & $1: 2000$ \\
\hline $\mathrm{pH}$ & & 6,6 & 7,2 \\
\hline Geruchsschwellenwert & & 200 & 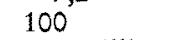 \\
\hline Geschmacksschwellenwert & & 50 Millionen & 20 Millionen \\
\hline Außere Beschaffenheit & & farblos, klar & farblos, klar \\
\hline Schwerflüchtige Kohlenwasserstoffe & $\mathrm{mg} / \mathrm{l}$ & 2,0 & 1,5 \\
\hline Leichtflüchtige Kohlenwasserstoffe & $\mathrm{mg} / 1$ & 15 & 8 \\
\hline Blei & $\mathrm{mg} / 1$ & 0,04 & 0,02 \\
\hline Phenole & $\mathrm{mg} / \mathrm{l}$ & 0,2 & 0,1 \\
\hline $\mathrm{KMnO}_{4}$-Verbrauch & $\mathrm{mg} / \mathrm{l}$ & 28 & 25 \\
\hline $\mathrm{K}_{2} \mathrm{Cr}_{2} \mathrm{O}_{7}$-Verbrauch & $\mathrm{mg} / \mathrm{l}$ & 304 & 240 \\
\hline $\mathrm{BSB}_{5}$ & $\mathrm{mg} / \mathrm{l}$ & 15 & 8 \\
\hline Aldehyde & $\mathrm{mg} / \mathrm{l}$ & n. n. & n. n. \\
\hline Jodzahl & $\mathrm{mg} / \mathrm{l}$ & 32 & 10 \\
\hline m-Alkalität & mval & - & 3,6 \\
\hline $\mathrm{CO}_{2}$ & $\mathrm{mg} / \mathrm{l}$ & 130 & $60^{\circ}$ \\
\hline $\mathrm{CO}$ & $\mathrm{mg} / \mathrm{l}$ & 40 & 20 \\
\hline
\end{tabular}

Zur Klärung der Frage, inwieweit die Abgase und Olrückstände von Außenbordmotoren eine schädliche Wirkung auf die pflanzlichen und tierischen Organismen im Gewässer haben, wurden von der biologischen Seite her Reihenversuche mit verschiedenen Fischarten im Labor durchgeführt, und zwar mit den im Tankversuch hergestellten Wässern. Diese bildeten die Grundlage für die späteren Teichversuche.

Es wurden Fischarten gewählt, die sich allgemein für Bioassays bewährt haben: Guppys, $\mathrm{K}$ arpfen $\left(\mathrm{K}_{1}\right)$ und Forell en $(10 \mathrm{~cm}$ lange Setzlinge).

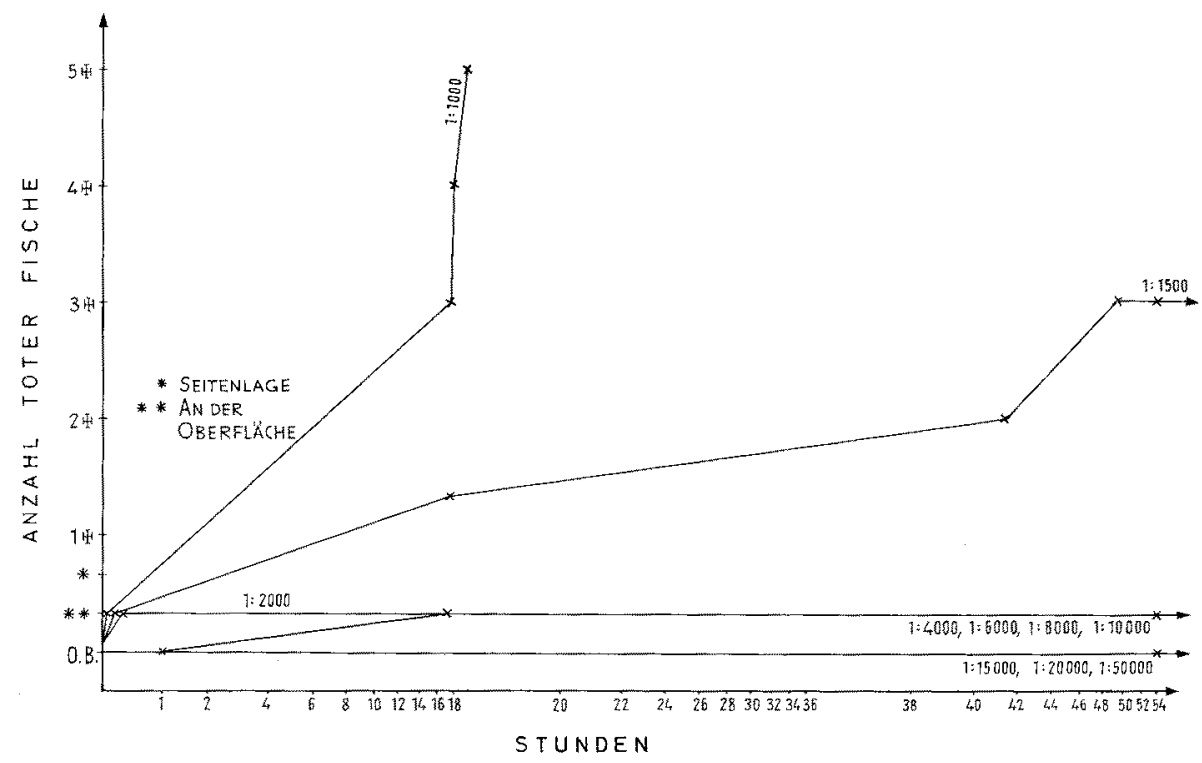

Abb. 1: Toxizitätsuntersuchungen an Guppys 
Für die erste Versuchsreihe wurde eine Grundkonzentration von 1:2000 benutzt und eine Verdünnungsreihe von $1: 2000 / 1$ : 4000/1 :6000/1 :8000/1 : 10000/1 : 15000/1 : 20000 und $1: 50000$ angesetzt. Wie aus Abbildung 1 hervorgeht, blieben die Guppys über 54 Stunden, teilweise bis 72 Stunden im Versuch. Die oben angeführten Konzentrationen zeigten keine schädigende oder gar tödliche Wirkung. Die Tiere standen jedoch fortwährend mit verstärkter Atemfrequenz an der Oberfläche.

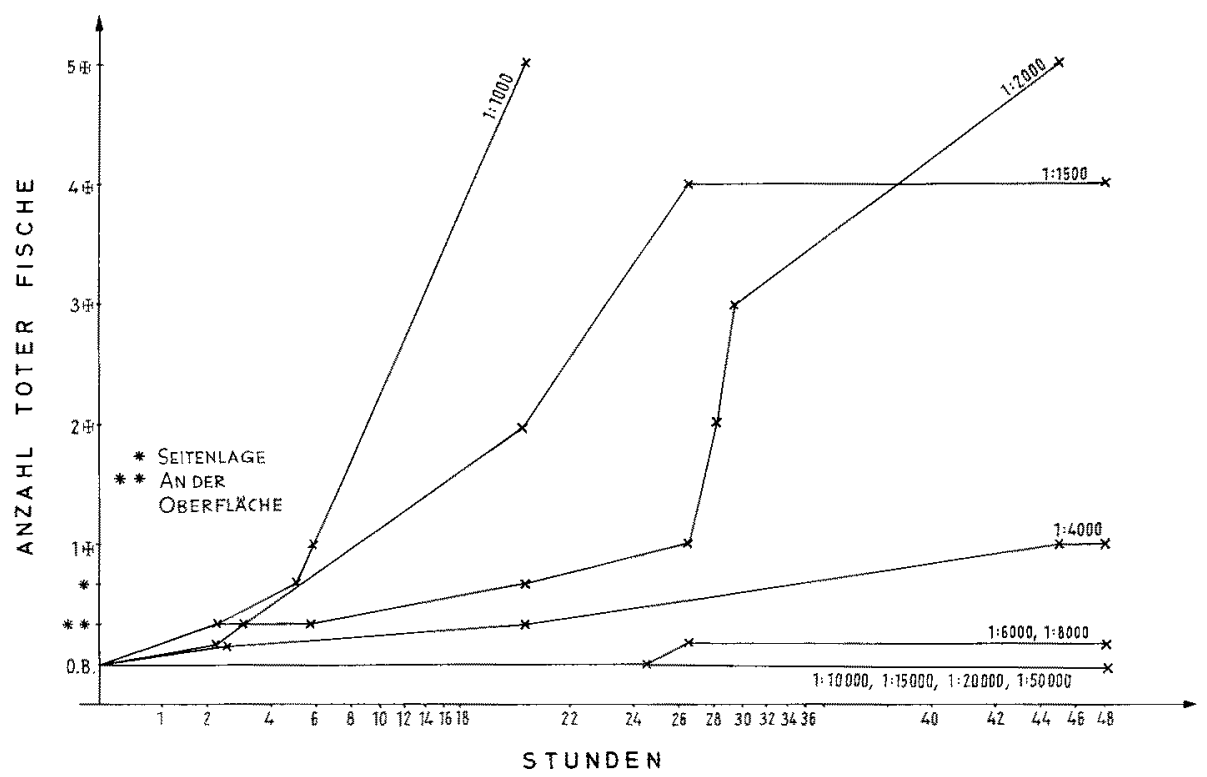

Abb. 2: Toxizitätsuntersuchungen an Karpfen $\left(K_{1}\right)$

Bei den $K$ a r p f e $n$ zeigte sich allerdings ein anderes Bild (Abb. 2). Hier begannen die Tiere bei der Konzentration $1: 2000$ schon nach ca. 2 Stunden an der Oberfläche zu hängen. Die Atmung wurde immer heftiger, später mehr verkrampft. Nach 21 Stunden nahm der erste Karpfen die Seitenlage ein, die Atmung wurde nun langsamer. Innerhalb von $26,28,30$ und $44 \mathrm{Std}$. gingen die Tiere nacheinander ein.

Auch bei der Konzentration $1: 4000$ stiegen die Karpfen an die Oberfläche. Ein Tier ging nach 44 Std. ein, die anderen wurden nach Ablauf der Versuchszeit in Frischwasser überführt und erholten sich sehr schnell. Die übrigen Konzentrationen zeigten keine weitere Beeinträchtigung der Fische mehr. Bei den Konzentrationen $1: 6000$ und $1: 8000$ gingen sie zwar an die Oberfläche, waren sonst aber ohne Befund. In den Becken mit den folgenden Konzentrationen schwammen sie völlig normal.

Die For elle n reagierten als sehr empfindliche Frischwassertiere in den Versuchen am schnellsten (Abb. 3). Bei der Konzentration $1: 2000$ nahmen sie schon nach 20 Min. die Seitenlage ein, nach 50 Min. waren bereits zwei Fische tot. Trotz Zeichen einer Schädigung (ruhiges Stehen an der Oberfläche bei verstärkter Atmung) überstand die dritte Forelle den Versuch. Ein ähnliches Bild, nur zeitlich etwas verschoben, zeigte sich bei der Konzentration von $1: 3000$. Die erste Forelle nahm nach $40 \mathrm{Min}$. die 
Seitenlage ein und war dann nach $75 \mathrm{Min}$. tot. Die übrigen Tiere starben nach $2 \mathrm{bzw}$. 16 Stunden. Bei der Konzentration $1: 4000$ ging die erste Forelle nach 21/4 Stunden ein; die beiden anderen Tiere standen zwar mit herabgesetzter Atemfrequenz an der Wasseroberfläche, überlebten aber den Versuch. Bei der Konzentration von 1:6000

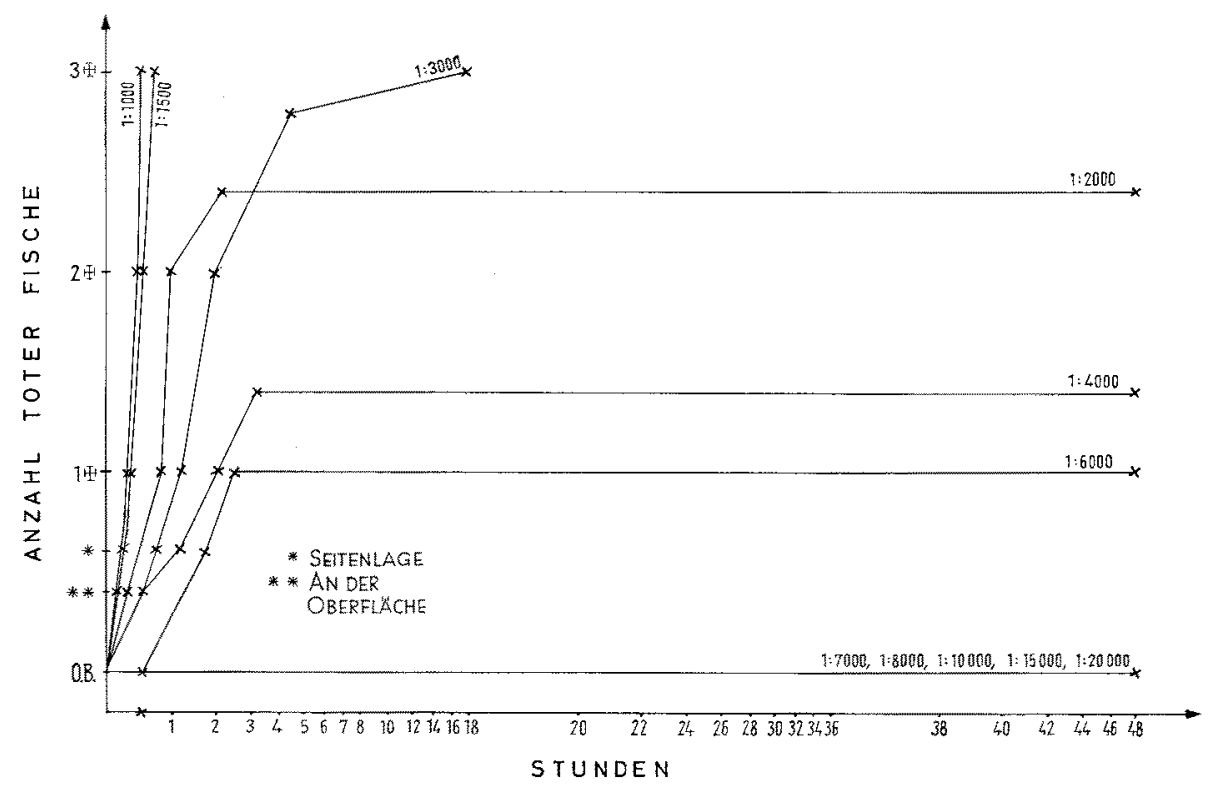

Abb. 3: Toxizitätsuntersuchungen an Forellen (Setzlinge)

zeigten die Forellen anfangs keine Reaktion, doch plötzlich (nach $100 \mathrm{Min}$.) ging ein Tier in die Seitenlage über und war nach weiteren $40 \mathrm{Min}$. tot. Die anderen Fische zeigten keinerlei Schädigungen. Auf die Konzentrationen 1:7000 bis 1:20000 reagierten die Fische dann in keiner Weise mehr negativ.

Zur Absicherung der Ergebnisse bezüglich der letalen Konzentration erhielten wir vom Institut für Verbrennungskraftmaschinen der Technischen Universität Berlin ein zweites Tankwasser von $1: 1000$, mit der wir einmal unverdünnt und zum zweiten bei einer Konzentration von $1: 1500$ arbeiteten. In den Versuchen mit der Konzentration $1: 1000$ gingen sowohl die $\mathrm{Guppys}$ wie auch die $\mathrm{K}$ a r p fen innerhalb von 18 bis 20 Stunden alle ein; die For e 11 en sogar schon nach $30 \mathrm{Min}$.

Die Toxizität der Konzentration $1: 1500$ trat für die $\mathrm{G}$ u p p y s und $\mathrm{K}$ a r pf e n nicht mehr so schlagartig zutage. Drei Guppys gingen nach 18, 41 und $48 \mathrm{Std}$. ein, zwei überlebten. Bei den Karpfen waren zwei nach $20 \mathrm{Std}$, einer nach $24 \mathrm{Std}$. tot. Die restlichen zwei erholten sich im Frischwasser der Nachbeobachtungsbecken sehr schnell. Für die For elle n war kaum ein Unterschied zu der Konzentration $1: 1000$ festzustellen. Die drei Tiere gingen innerhalb von $30 \mathrm{Min}$. ein.

Nach unseren Untersuchungen ist demzufolge die Konzentration $1: 1000$ als die für die Guppys letale Konzentration anzusehen. Bei den Karpfen wirkten die Konzentrationen $1: 1500$ und $1: 2000$ noch ähnlich toxisch, wenn auch 
erst nach längerer Zeit. Für die For elle $\mathrm{n}$ ist die Konzentration $1: 3000$ noch als stark giftig zu bezeichnen.

\section{Chemische Analyseder Tankwässer}

Betrachtet man die chemische Analyse der Tankwässer bezüglich der Fischtoxizität, so interessieren hier folgende Punkte: der Gehalt an leicht- und schwerflüchtigen Kohlenwasserstoffen, Blei, Phenolen, $\mathrm{CO}$ und $\mathrm{CO}_{2}$.

Die Einwirkung von leichten und schweren Kohlenwasser$s$ t of $f e n$ ist allgemein recht schwierig zu beurteilen, da es sich um Kohlenwasserstoffgemische handelt, die für eine genaue Beurteilung ihrer Giftigkeit im einzelnen schlecht faßbar sind. In der Literatur findet man nur wenige Angaben. über Versuche mit Benzinen und Olen (ZahNer 1962, LIEBMANN 1958), wobei auch darauf hingewiesen wird, daß oftmals nicht nur die reinen Stoffe selbst, sondern die verschiedenen Zusatzstoffe (z. B. Autobenzine) und auch die Krackprodukte die Giftwirkung verstärken.

Bei überwiegend niedermolekularen Kohlenwasserstoffverbindungen können Fluchtreaktionen ausgelöst werden. Falls jedoch höhermolekulare Fraktionen vorliegen, kommt es in der Regel zu einer zunehmenden Aktivitätsminderung, die in völlige Bewegungsunfähigkeit übergehen kann.

Als letale Grenzkonzentration für Benzin wird die Menge von $50 \mathrm{mg} / 1,100 \mathrm{bis} 260 \mathrm{mg} / 1$ Autobenzin normal und 40 bis $100 \mathrm{mg} / \mathrm{l}$ Autobenzin super angegeben.

Für Ole liegen die entsprechenden Werte zwischen 50 und $500 \mathrm{mg} / 1$ Wasser. Da die chemische Analyse der Tankwässer einen Gehalt an schweren Kohlenwasserstoffen von $2,0 \mathrm{mg} / 1$ (bei der Konzentration $1: 1000$ ) und 1,5 mg/l (bei der Konzentration $1: 2000)$ ergab, bzw. an leichten Kohlenwasserstoffen von $15 \mathrm{mg} / 1(1: 1000)$ und $8 \mathrm{mg} / \mathrm{l}$ (1 2000), kann man hier kaum von einer Schädigung a 11 e in durch die Kohlenwasserstoffe sprechen.

B leive r bin d u ng e n können für Fische sehr schädlich sein. Sie lagern sich über eine lange Zeitdauer hin im Körper, hauptsächlich aber in den Kiemen ab und können so zu "schleichenden" Vergiftungen führen. Allerdings liegen die im Versuch gefundenen Werte (nach LiEBMANN [1958] 0,2 bis $10 \mathrm{mg} / \mathrm{l}$ ) weit über denen der vorliegenden Analyse $(0,04 \mathrm{mg} / 1$ bei $1: 1000$ und 0,02 bei $1: 2000)$.

Die schädliche Wirkung von $\mathrm{Ph}$ e nol en richtig einzuschätzen, ist ähnlich schwierig, wie die der Kohlenwasserstoffe; da sie in der Praxis nur in Gegenwart anderer giftiger Stoffe vorkommen. So in den Abwässern von Gaswerken, Kokereien (Gasabwässer), ferner bei der Stein- und Braunkohlenschwelerei usw. Die für Fische tödlichen Werte liegen hier bei 6 bis $17 \mathrm{mg} / 1$, also 30 - bis 80 fach höher, als die chemische Analyse der Tankwässer ergab (0,2 bis $0,1 \mathrm{mg} / 1)$.

Ober die Wirkung von Kohle $\mathrm{nm}$ onoxid auf Wassertiere liegen in der Literatur Berichte über einige Fischarten sowie Krebse vor. Nach Untersuchungen von Wells (1918) mit verschiedenen amerikanischen Fischarten töten 1,5 mg/1 Jungfische in 1 bis 6 Stunden, $7,5 \mathrm{mg} / 1$ in $28 \mathrm{Min}$. bis $5^{1 / 2} \mathrm{Std}$. und $14,6 \mathrm{mg} / \mathrm{l}$ in $10 \mathrm{Min}$. bis 
4 Std. die Tiere ab. Nach PaAsch (1901) gingen bei $1,5 \mathrm{mg} / \mathrm{l}$ die Fische nach $10 \mathrm{Min}$. in die Rückenlage über und waren nach $1 \frac{1}{1 / 2}$ Std. tot.

Kohlendioxid ist im Wasser gut löslich und reagiert mit diesem nur zu einem sehr geringen Prozentsatz zu Kohlensäure. In Wasser eingeleitetes $\mathrm{CO}_{2}$ wirkt daher nicht wie eine Säure durch pH-Erniedrigung für die Fische toxisch, sondern schädigt durch seinen hohen Gehalt an $\mathrm{CO}_{2}$-Molekülen. Erhöhter $\mathrm{CO}_{2}$-Druck zwingt die Fische ähnlich wie bei Sauerstoffmangel zu einer Notatmung (Stehen an der Wasseroberfläche verbunden mit hettigen Atembewegungen), da das $\mathrm{CO}_{2}$ hauptsächlich auf das Atemzentrum einwirkt und die Affinität des Blutes gegenïber $\mathrm{O}_{2}$ senkt. Auffallend ist, daß bei Erstickungen durch $\mathrm{O}_{2}-$ Mangel die Kiemendeckel weit abgespreizt sind, bei Vergiftung durch $\mathrm{CO}_{2}$ aber eng anliegen. Letzteres Symptom konnte auch bei unseren Versuchen festgestellt werden.

Wie amerikanische Versuche ergaben (zitiert nach LIEBMANN 1958) wirkten $\mathrm{CO}_{2}$ Mengen von 70 bis $100 \mathrm{mg} / \mathrm{l}$ für eine Anzahl untersuchter Fischarten in 33 bis $150 \mathrm{Std}$. tödlich. Teilweise sollen sogar schon $20 \mathrm{mg} / 1$ stark toxisch gewesen sein. Nach HAUpT (1932) liegt die letale Konzentration für Karpfen und Schleie bei $200 \mathrm{mg} / \mathrm{l}$, für Forellen bei $45 \mathrm{mg} / 1$.

Die chemische Analyse der Tankwässer ergab einen CO-Gehalt von $40 \mathrm{mg} / \mathrm{l}$ (bei $1: 1000)$ und $20 \mathrm{mg} / \mathrm{l}$ (bei $1: 2000)$ sowie einen $\mathrm{CO}_{2}$-Gehalt von $130 \mathrm{mg} / \mathrm{l}(1: 1000)$ und $60 \mathrm{mg} / 1(1: 2000)$. Vergleicht man diese Werte mit den obigen Angaben, so ergibt sich, daß der $\mathrm{CO}$ - und $\mathrm{CO}_{2}$-Gehalt hauptsächlich für die Toxizität der Tankwässer verantwortlich zu machen ist. Interessant ist in diesem Zusammenhang noch, daß CO von den Fischen kaum wahrgenommen wird, $\mathrm{CO}_{2}$ jedoch Fluchtreaktionen auslöst.

Während $\mathrm{CO}$ und $\mathrm{CO}_{2}$ in größeren Konzentrationen im Wasser gasförmig gelöst sind und als Atemgift toxisch wirken, verändern die anderen Verbrennungsprodukte (z. B. leicht- und schwerflüchtige Kohlenwasserstoffe, Blei, Phenol) bereits in verhältnismäßig geringer Konzentration durch Anreicherung und Speicherung das Fischfleisch in seinem Geschmack.

\section{Teichversuche}

Nach Abschluß der Brems- und Tankversuche, in denen die erforderlichen Grundlagen erarbeitet worden waren, sollten in einem Versuchsteich von etwa $170 \times 25 \mathrm{~m}$ und etwa $2,0 \mathrm{~m}$ Tiefe die praktischen Verhältnisse wie auch die meteorologischen Einflüsse möglichst genau berücksichtigt und erfaßt werden. Außerdem stand noch ein angrenzender kleinerer Kontrollteich zur Verfügung.

Ein mit dem Außenbordmotor II (20 PS, mittlere Leistungsklasse Mischungsverhältnis $1: 50$ ) ausgerüstetes Kunststoffboot hatte ein bestimmtes Fahrprogramm wöchentlich zu absolvieren, wobei die verbrauchte Brennstoffmenge und der Pegelstand laufend registriert wurden.

Von den chemischen Ergebnissen sind vor allem interessant der Geruchs$\mathrm{sch}$ welle $\mathrm{n}$ wer $\mathrm{t}$, der durch entsprechende Verdünnung der Proben mit geruchfreiem Wasser ermittelt und von mehreren Versuchspersonen geprüft wurde. Er zeigt sehr eindeutig die steigende Belastung des Gewässers während der Versuchszeit. Als 
Spezifikum konnte bereits kurz nach Versuchsbeginn ein scharfer brenzliger mineralölartiger Geruch festgestellt werden, der beim Fischtest geschmacklich auch wieder auftrat. Interessant ist ein Vergleich der aus Schöpf- und Sammelproben gefundenen Werte. Danach lagen die Ergebnisse der ersteren vielfach um 100\% höher, was dafür spricht, daß in erster Linie die obersten Wasserschichten von der Verunreinigung betroffen sind. Dies mag auch eine Frage der Durchmischung sein, die nach der Tiefe zu abnimmt.

Der Gehalt an schwerflüchtigen Kohlenwasserstoffen im Gewässer nimmt anfangs langsam und stetig zu, wenn auch die absoluten Werte sehr günstig sind und maximal bei $1 \mathrm{mg}$ OI/l Wasser liegen. Charakteristisch sind auch hier die steileren Anstiege während der eigentlichen Versuchstage der Woche, an denen gefahren wurde. Gegen Versuchsende (Anfang November) fallen die Werte wieder ab. Der Vergleichsteich weist dagegen einen fast gleichmäßig niedrigen Olgehalt auf, der, wie beim Versuchsteich, auf die Verbindung des Teufelssee-Stichkanals mit der Havel vor der Erstellung der Spundwand zurückzuführen sein dürfte.

Wie bereits erwähnt, ist Mineralöl i. a. wenig wasserlöslich (hydrophob) und meistens als Emulsion im Wasser verteilt. Infolge der Befahrung werden diese Olfilme durcheinandergewirbelt und durch Wellenbewegung an Uferrändern, Baumwurzeln und Pflanzen teilweise niedergeschlagen. Von einer zusammenhängenden Olschicht kann keine Rede sein. Neben dieser rein mechanischen Verteilung wird das Ol im Laufe der Zeit noch einer chemisch-biologischen Veränderung unterworfen sein. Die verharzten und teils agglomerierten Olteilchen werden dann in den Untergrund gelangen, wo sie im Bodenschlamm festgehalten werden. Wie Schlammanalysen des Versuchsteichs zeigten, war tatsächlich eine geringe Zunahme der Gehalte an schwerflüchtigen Kohlenwasserstoffen von $0,5 \%$ auf $0,9 \%$ bezogen auf Trockensubstanz in Schlamm während der Versuchszeit festzustellen. Eine Identifizierung dieser Stoffe als Mineralöl mittels Dünnschichtchromatographie war möglich. Auf Grund der Tankversuche wäre bei einem Verhältnis von verbrauchtem Brennstoff: Wasser von 1:3000, wie es bei Versuchsende vorlag, ein Gehalt an schwerflüchtigen Kohlenwasserstoffen von etwa $0,7 \mathrm{mg} / \mathrm{L}$ zu erwarten gewesen, was auch etwa in der Größenordnung der beim Teichversuch gefundenen Werte liegt.

Die $S$ a u e r s off gehalte der Gewässer liegen verhältnismäßig tief und entsprechen etwa 50\% der jeweiligen Temperatur-Sättigungswerte. Eine geringe periodische Schwankung der Sauerstoffgehalte dürfte durch die Belüftung des Gewässers infolge seiner Befahrung zu erklären sein.

Zur morphologisch-hydrobiologischen Charakterisierung der Teiche sei folgendes gesagt: der Versuchsteich mit einer Ausdehnung von etwa $170 \times 25 \mathrm{~m}$ war dicht von Bäumen, zumeist Erlen, umstanden und demzufolge die größte Zeit des Tages über stark beschattet. Entsprechend dicht war auch im Herbst der Laubfall, der zu einer Huminstoffanreicherung des Wassers und zur Bildung einer stärkeren Schlammschicht beitrug.

Innerhalb der Uferzone wuchsen teilweise große Bestände von Myriopbyllum verticillatum und Nymphaea candida, an den Uferrändern vereinzelte Gruppen von Acorus calamus, Iris pseudacorus, Bidens tripartitus, Lycopus europaeus $u$. a. Der Kontrollteich östlich der Straße bot ein ähnliches Bild, nur zog sich an der einen Längs- 
seite ein ca. $10 \mathrm{~m}$ langer "Sandstrand“ hin, der im Wasserbereich von Muscheln (Unio und Anodonta) besiedelt wurde. Im Unterschied zum Versuchsteich lag im Kontrollteich die Temperatur durch den Kühlwasserauslauf des $200 \mathrm{~m}$ entfernt gelegenen Kraftwerkes Oberhavel um einige Grade höher. Nach dem Setzen der Spundwand glich sich jedoch die Temperatur der des Versuchsteiches an. Ferner war je nach der Windrichtung die Oberfläche des Kontrollteiches mit einer dünnen Flugaschenschicht bedeckt, deren Ursprung gleichfalls das Kraftwerk war. Die biologischen Wasserproben (Plankton, Bewuchs und Grund) wurden parallel zu den chemischen Proben genommen. In seiner Zusammensetzung glich das Plankton weitgehend dem der Havel. Die Auswertungen der Proben ergaben das folgende hydrobiologische Bild der beiden Teiche.

Im Versuchsteich traten, neben vereinzelten Oscillatorien, hauptsächlich L y n g byen auf, die ihre größte Verbreitung in den Herbst- und Wintermonaten hatten. Die Hauptmenge der $\mathrm{K}$ i eselalgen wurde im Frühjahr von Fragilaria capucina, Synedra acus, Eunotia valida und Navicula gestellt. Im Sommer ging die Individuenzahl dieser Arten allmählich zurück, um dann in das typische Winterplankton, zusammengesetzt aus Asterionella formosa und Synedra ulna, überzugehen.

Von den Grünalgen traten im Bewuchs hauptsächlich Spirogyra spec. im späten Frühjahr auf; Oedogonium und Cladophora konnten ab August bis in den Winter hinein festgestellt werden.

Bei den tierischen Organismen fiel im Frühjahr 1965 das massenhafte Vorkommen von Dinobryon sertularia auf. Von Juli bis September setzte dann eine starke Vermehrung von Volvox aureus ein, die im März 1966, also nach Beendigung der Versuche, von einer Mallomonas-Euglena acus-Population abgelöst wurde.

Von den Ciliaten traten, neben kleineren, beweglichen Formen, hauptsächlich im Bewuchs die festsitzenden Koloniebildner, wie Carchesium und Vorticella, auf. Sie hatten ihre maximale Verbreitung im Herbst und Winter.

Für die Rädertierchen (Rotatorien) waren die starken Populationen von Keratella cocblearis, $K$. quadrata, Polyartbra platyptera und Asplanchna priodonta vor dem Beginn der Versuche auffällig.

Von den Krebstieren traten die Cyclopiden und ihre Vermehrungsstadien ( $\mathrm{N} \mathrm{a} \mathrm{u} \mathrm{-}$ pli e n) am häufigsten auf. Sie konnten hauptsächlich vom Herbst bis zum späten Frühjahr festgestellt werden. Andere Arten, wie z. B. Diaphanosoma brachyurum, traten nur innerhalb ganz kurzer Perioden auf.

Der Kontrollteich besaß im Vergleich zum Versuchsteich einige Unterschiede in seinem Organismenbestand sowie Abweichungen innerhalb der jahreszeitlichen Verteilung des maximalen Auftretens der einzelnen Populationen.

Bei den $B 1$ a u a $1 \mathrm{~g}$ e $\mathrm{n}$ war der augenfälligste Unterschied zum Versuchsteich zu vermerken. Hier bildeten nicht die Oscillatorien und Lyngbyen die hauptsächlichsten Vertreter, sondern die an der Wasseroberfläche flottierenden Arten Microcystis aeruginosa und Aphanizomenon flos-aquae. Sie vermehrten sich in den Sommermonaten so stark, daß es zu einer Wasserblüte kam.

Die Kieselalgen, mit den Arten Melosira granulata, Gomphonema ventricosum, Cocconeis pediculus und Diatoma vulgare, bildeten im Frühjahr 1965 eine Massenentwicklung, die sich aber im Gegensatz zum Versuchsteich über das ganze Sommerhalbjahr halten konnte und im Herbst und Winter noch durch die für diese Jahreszeit 
typischen Vertreter (Asterionella formosa, Synedra ulna, S. acus und Diatoma elongatum) verstärkt wurde.

Die beim Versuchsteich genannten Grünalgen zeigten im Kontrollteich im Spätherbst und Winter ein bedeutend stärkeres Wachstum.

Bei den tierischen Organismen traten innerhalb der Flagellaten hauptsächlich Chlamydomonaden auf, und zwar mehr oder weniger gleichmäßig über das Jahr verteilt.

Die Ciliaten des Kontrollteiches setzten sich mehr aus den freibeweglichen Formen zusammen. Die koloniebildenden Gattungen, wie Vorticella, Carchesium und Epistylis, traten dagegen sehr zurück.

Die Populationen der Rädertiere wiesen die gleiche Artenzusammensetzung auf, die Vermehrung jedoch und das jahreszeitliche Auftreten war im Vergleich zum Versuchsteich unterschiedlich. Bei den Crustaceen traten neben den bereits erwähnten Cyclopiden die Arten Diapbanosoma brachyurum, Polyphemus pediculus und Ceriodaphnia quadrangula auf. Sie waren das ganze Jahr über gleichmäßig häufig verteilt.

Wie sich bei einem Vergleich der beiden Teiche gezeigt hat, traten in der $\mathrm{Zu}$ sammensetzung, der Menge und der jahreszeitlichen Verteilung der Wasserorganismen Unterschiede zutage.

Bei den Kiesel- und Grünalgen sowie den Rotatorien und Krebstieren ergab sich eine stärkere Abnahme der Populationen und Arten nach Beginn der Versuche und ein allmähliches Erholen nach Beendigung derselben im Herbst. Das schnelle Zurücktreten der oben genannten Organismen nach Beginn der Motorbootversuche kann als Anzeichen einer zunehmenden Belastung gewertet werden. An den submersen Wasserpflanzen und der Ufervegetation konnte keine negative Wirkung durch Benzine oder Ole festgestellt werden.

\section{Geschmacksuntersuchungen an Fischen}

$\mathrm{Z}$ us a m m e n f a s s en d kann gesagt werden, daß noch keine pflanzlichen und tierischen Wasserorganismen als Indikatoren für Benzine und Ole bekannt sind und deshalb noch keine genaue Beurteilung nach den bisher üblichen Methoden der ökologischen Wassergütebestimmung gegeben ist. Eine Schädigung bestimmter Wasserorganismen ist durchaus möglich.

Von besonderer Bedeutung für das Gesamtproblem schien von vornherein die geschmadkliche Beeinflussung des Fischfleisches zu sein, die in sogenannten Fischgeschmackstesten untersucht wurden. Hierzu wurden mit Beginn der Versuche an jedem Wochenanfang eine Anzahl von Testpersonen gestellt, die drei Fische verschiedener Herkunft geschmacklich prüfen mußten. Als geschmacksneutrale Fischart wurden Forellen aus einer Züchterei gewählt. Die Testfische wurden vorher aus dem Versuchsteich geangelt. Es handelte sich dabei hauptsächlich um Plötzen, Rotfedern, kleinere Barsche, Güstern und Schleie. Die Fische wurden in getrennten Töpfen gekocht, die einzelnen Kostproben mit je einer Codezahl numeriert und den Testpersonen vorgesetzt. Auf einem Fragebogen standen drei Geschmacksangaben „ohne Befund“, "modrig" und 
„ölig“ (nach Verbrennungsrückständen) und die Geschmadksnuancen kein Olgeschmack, leicht ölig, ölig, starkölig und ungenießbar zur Verfügung.

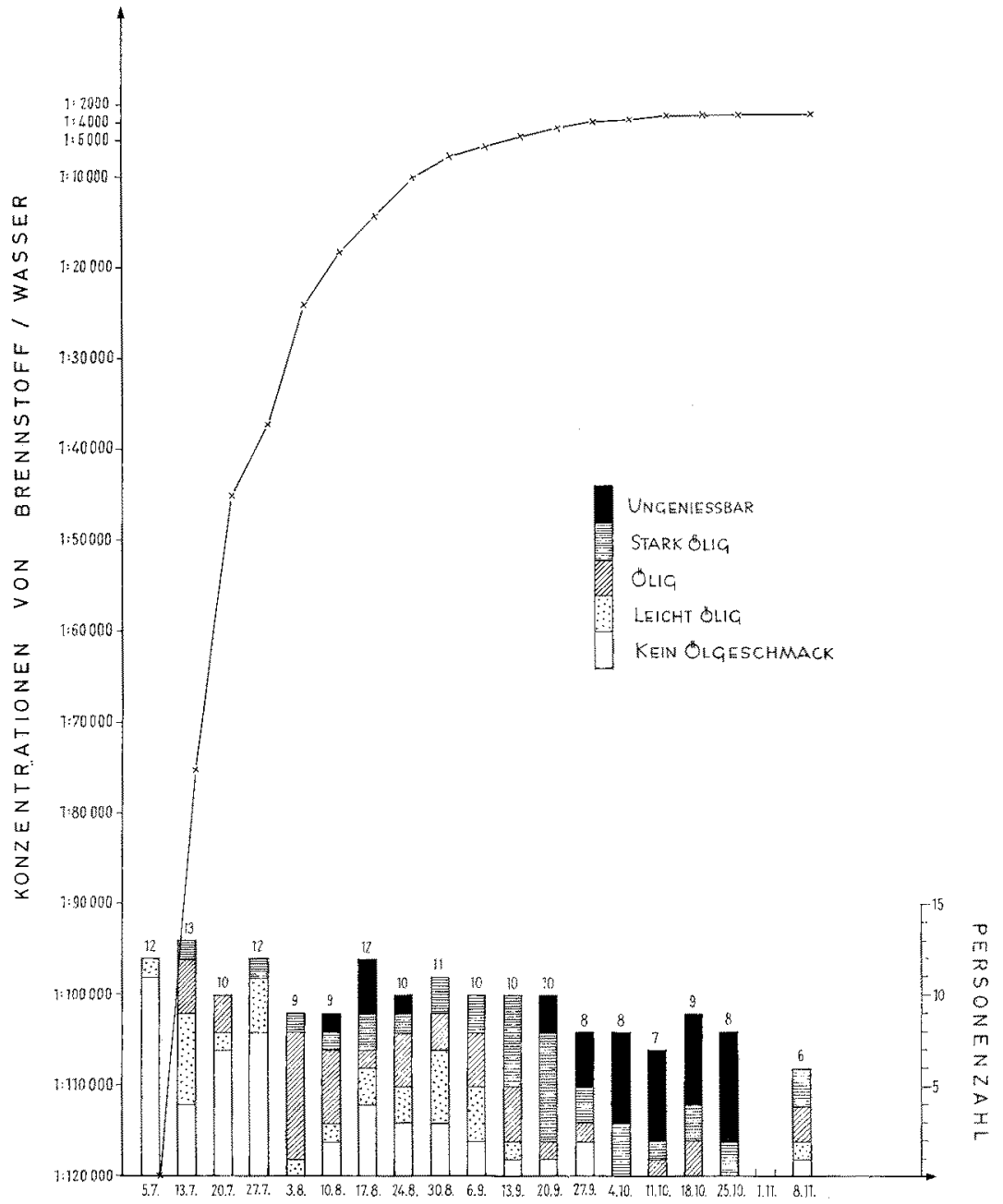

Abb. 4: Tests zur Prüfung einer geschmadklichen Beeinflussung des Fischfleisches

Die ersten Testpersonen stellten schon nach gut einer Woche einen brenzlig-öligen Geschmack bei den Fischen des Versuchsteiches fest. Die zu diesem Zeitpunkt erreichte Konzentration Brennstoff/Wasser betrug ungefähr 1:75000. Nach einem Monat waren die Fische für eine Anzahl von Personen bereits ungenießbar (bei 1:25000). Diese geschmackliche Feststellung nahm dann mit steigender Konzentration im Versuchsteich stark zu. Die entstandene Lücke im Bereich „ungenießbar“ (vom 30. August bis 13. September) ist auf die Urlaubszeit und die damit verbundene laufende „Neueinstellung" von Testpersonen zurückzuführen. Allerdings ist die Geschmacksangabe „stark ölig" hier prozentual bedeutend höher als vorher (Abb. 4). 
Der vergleichsweise durchgeführte Geschmackstest bei den Fischen des Kontrollteiches zeigt, daß der größte Teil der Personen während der ganzen Versuchsdauer keinen öligen Geschmack feststellte. Außerdem konnte noch das Kochwasser, das einen deutlichen Geruchsunterschied zu dem vorigen aufwies, als Testmittel herangezogen werden (Abb. 5).

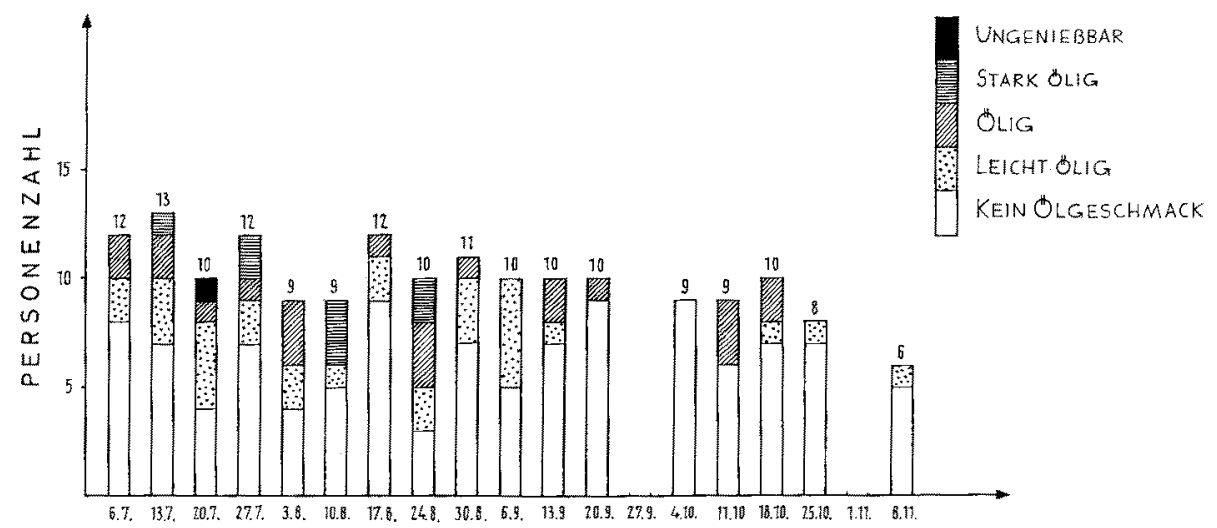

Abb. 5: Kontrollversuche zu den Geschmackstests im Kontrollteich (vgl. Abb. 4)

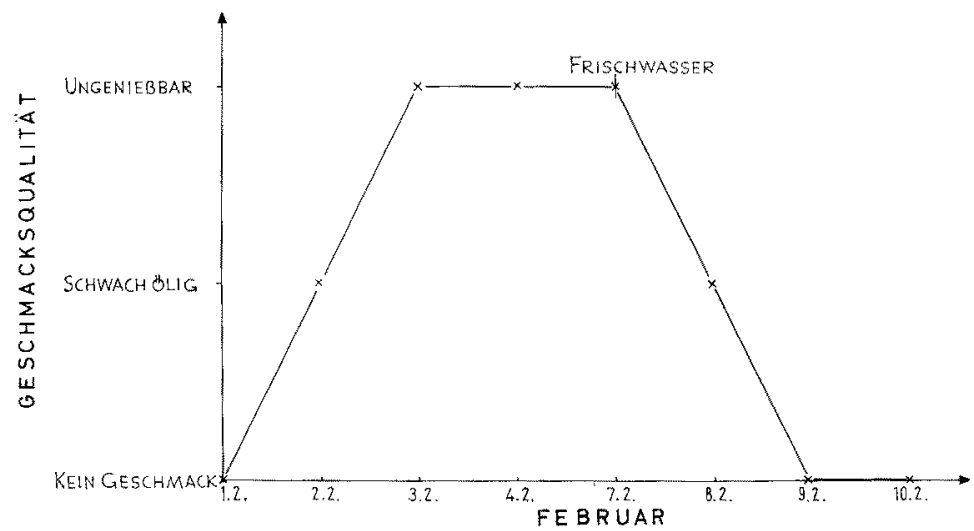

Abb. 6: Versuche zur Geschmacksbeeinträchtigung durch Verbrennungsrückstände (Konzentration: $1: 2000$ ) bzw. Geschmacksverbesserung von Karpfenfleisch durch Aufenthalt in Frischwasser

Die Speicherung der Verbrennungsrückstände in den Fischen fand nach unseren Beobachtungen hauptsächlich im D a rmfett statt. Die Rücken- und Schwanzmuskulatur schmeckte bedeutend weniger nach Benzinrückständen als die $\mathrm{B}$ a u ch 1 a p p e n. Es konnte jedoch nicht festgestellt werden, daß die im Versuchsteich vorkommenden Fischarten durch die Verbrennungsrückstände sichtbar geschädigt oder getötet wurden.

Um noch genauere Angaben darüber zu erhalten, wie schnell Verbrennungsrückstände bei Fischen gespeichert werden, wurden im Labor eine Anzahl von Karpfen in 
einer Tanklösung von 1:2000 gehältert. In einem Zeitraum von 10 Tagen wurde ein Fisch pro Tag entnommen, gekocht und auf seinen Geschmack hin geprüft. Aus Abbildung 6 ist $\mathrm{zu}$ ersehen, daß der Beginn der Geschmacksbeeinträchtigung sehr früh einsetzte, daß sich aber auch nach dem Uberführen der Fische in Frischwasser der brenzlig-ölige Geschmack ziemlich schnell verlor.

Die Ergebnisse der Freilandversuche zeigten, daß die unter praxisnahen Versuchsbedingungen erhaltenen Konzentrationen an Verbrennungsrückständen auf jeden Fall bei den Fischen eine Geschmacksbeeinflussung herbeiführen. Das kann sich auf wirtschaftlichem Sektor derart auswirken, daß ganze Fänge ungenießbar sind. Darüber hinaus muß man noch berïcksichtigen, daß durch eine mögliche Beeinträchtigung des Planktons der Fischwelt auch die Nahrungsbasis entzogen werden kann. Die Tatsache, daß die im Versuchsteich erreichte Konzentration $1: 3000$ nicht wie in den Tankversuchen zu einem Fischsterben führte, beruht sicherlich darauf, daß hier durch das Befahren mit dem Motorboot die leicht flüchtigen toxischen Bestandteile, insbesondere $\mathrm{CO}$ und $\mathrm{CO}_{2}$ verhältnismäßig schnell aus dem Wasser entweichen.

\section{ZUSAMMENFASSUNG}

1. An der Verschmutzung der Gewässer durch Mineralöle und deren Produkte sind in zunehmendem Maß auch die Wasserfahrzeuge mit Außenbordmotoren, gemischgeschmierten Zweitaktern, beteiligt. Da aber die Oberflächengewässer immer stärker auch für die Trinkwassergewinnung herangezogen werden müssen, sind die Untersuchungen auch von allgemeinhygienischem Interesse.

2. Die durchgeführten Versuche, in denen in Abhängigkeit von Motorleistung und Mischungsverhältnis (Schmieröl - Brennstoff) die anfallenden Abgas-, Ol- und Krackprodukte chemisch untersucht worden sind, ließen einen Vergleich mit Zahlenangaben anderer Autoren zu. Der Anfall an Schmieröl, das wegen seiner schlechten biochemischen Abbaubarkeit besonders gravierend ist, kann allerdings durch Verwendung eines Brennstoffgemisches von $1: 50$ statt wie üblich $1: 16$ bis $1: 25 \mathrm{um}$ etwa $50 \%$ herabgesetzt werden.

3. Für die Tankversuche wurden durch Einleiten der Motorauspuffgase in einen wassergefüllten Behälter Versuchswässer in Konzentrationen von 1:1000 und $1: 2000$, d. h. Kraftstoffverbrauch zu Wasser mit Motor II (Nennleistung 18 PS, Mischungsverhältnis $1: 50$ ) hergestellt und chemisch untersucht. Gleichzeitig wurden mit diesen konzentrierten Wässern orientierende Toxizitäts- und Geschmacksteste mit Fischen durchgeführt. Hierbei war die letale Dosis für Karpfen und Forellen sehr unterschiedlich. Aquarienversuche mit einem Tankwasser von 1:2000 zeigten, daß sich die geschmackliche Beeinflussung des Fischfleisches verliert, wenn nach einigen Tagen der Exposition die Versuchstiere wieder in Frischwasser gehalten werden.

4. Bei den Teich versuch en wurde durch den Betrieb eines Außenbordmotorbootes (20 PS) das Versuchsgewässer in steigendem Maße durch Auspuffgase belastet. Hierbei waren insbesondere Fische ein brauchbarer und empfindlicher Indikator für den Grad der Gewässerverölung, da sie infolge Speicherung der im Wasser gelösten, emulgierten und suspendierten Verbrennungsprodukte verhältnismäßig 
schnell geschmacklich ansprachen. Dadurch konnten auch unterschwellige Konzentrationen der Auspuffgase erfaßt werden. Während der Versuchszeit war bei steigendem Verbrauch an Kraftstoffgemisch eine zunehmende Belastung an organischen Stoffen festzustellen. Dies drückte sich in einem Anstieg des $\mathrm{KMnO}_{4}$-Verbrauches, des Geruchsschwellenwertes, im Gehalt an schwerflüchtigen Kohlenwasserstoffen und den Sauerstoffwerten aus; auch der Geschmack des Fischfleisches wurde von mehreren Versuchspersonen immer stärker als ölig bzw. ungenießbar empfunden.

5. Wie dargelegt werden konnte, ist eine irreparable Verölung von Gewässern durch den Betrieb von Außenbordmotoren nicht zu erwarten, wenn der Bootsbetrieb während der Saison mit der Größe des Gewässers und seinem biologischen Selbstreinigungsvermögen abgestimmt wird. In dieser Hinsicht kann auch einiges von der motortechnischen Seite aus getan werden, wie beispielsweise die Herabsetzung des Schmierölanteils im Brennstoffgemisch und eine ordnungsgemäße Wartung der Motoren.

6. Die Untersuchungen bezogen sich auftragsgemäß auf die Gewässerverschmutzung durch Außenbordmotoren, doch sind in diesem Zusammenhang die bei der Binnenschiffahrt anfallenden Bilgewässer- und Altöle zu erwähnen, die oft u n k on $\mathrm{t} r o 11$ i e $\mathrm{t}$ über Bord abgelassen werden. Diesem Obelstand wäre aber durch gesetzliche Vorschriften und Einrichtung von Annahmestellen für obige Olabfälle entlang den Wasserstraßen zu begegnen, wie es am Rhein und an der Elbe bereits praktiziert wird. Gerade im Hinblick auf die Bedeutung des Bodensees als Trinkwasserspeicher wird man hier die Entwicklung der Klein- und Binnenschiffahrt besonders sorgfältig beobachten und gegebenenfalls durch entsprechende Maßnahmen lenken müssen.

\section{ZITIERTE LITERATUR}

English, J. N., McDermott, G. N. \& Henderson, C., 1961. Können die Auspuffgase von Außenbordmotoren das Wasser merklich verschmutzen? U.S. Department of Health, Education and Welfare, Washington, D.C.

- Surber, E. W. \& McDermotT, G. N., 1961. Untersuchungen über den Anteil der Verschmutzung durch die Auspuffgase von Außenbordmotoren. U.S. Department of Health, Education and Welfare, Washington, D.C.

Haupt, H., 1932. Fischsterben durch saures Wasser. Vom Wass, 6, 261-262.

LrebmanN, H., 1960. Handbuch der Frischwasser- und Abwasserbiologie. Oldenbourg, München, Bd 2, 1-1149.

PaAsch, K., 1901. Einwirkung des Kohlenoxids auf Kaltblüter. Würzburg, Nat.-math. Diss. v. 1901.

WeLLS, M. M., 1918. The reactions and resistance of fishes to carbon dioxide and carbon monoxide. Bull. Ill. St. Lab. nat. Hist. 11, 557-571. 